Article

\title{
Phased Haplotype Resolution of the SLC6A4 Promoter Using Long-Read Single Molecule Real-Time (SMRT) Sequencing
}

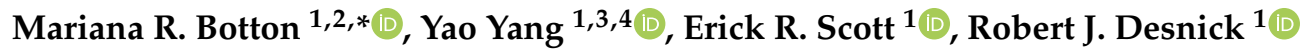 \\ and Stuart A. Scott $1,3,4,5, *$ (D) \\ 1 Department of Genetics and Genomic Sciences, Icahn School of Medicine at Mount Sinai, \\ New York, NY 10029, USA; yaoy@stanford.edu (Y.Y.); erick.scott@mssm.edu (E.R.S.); \\ robert.desnick@mssm.edu (R.J.D.) \\ 2 Cells, Tissues and Genes Laboratory, Hospital de Clínicas de Porto Alegre, Porto Alegre 90035-903, RS, Brazil \\ 3 Department of Pathology, Stanford University, Palo Alto, CA 94305, USA \\ 4 Stanford Medicine Clinical Genomics Program, Stanford Health Care, Stanford, CA 94305, USA \\ 5 Sema4, Stamford, CT 06902, USA \\ * Correspondence: mrbotton@hcpa.edu.br (M.R.B.); sascott@stanford.edu (S.A.S.); \\ Tel.: +55-51-3359-8852 (M.R.B.); +1-650-724-0973 (S.A.S.)
}

Received: 18 October 2020; Accepted: 10 November 2020; Published: 12 November 2020

\begin{abstract}
The SLC6A4 gene has been implicated in psychiatric disorder susceptibility and antidepressant response variability. The SLC6A4 promoter is defined by a variable number of homologous 20-24 bp repeats (5-HTTLPR), and long (L) and short (S) alleles are associated with higher and lower expression, respectively. However, this insertion/deletion variant is most informative when considered as a haplotype with the rs 25531 and rs 25532 variants. Therefore, we developed a long-read single molecule real-time (SMRT) sequencing method to interrogate the SLC6A4 promoter region. A total of 120 samples were subjected to SLC6A4 long-read SMRT sequencing, primarily selected based on available short-read sequencing data. Short-read genome sequencing from the 1000 Genomes $(1 \mathrm{KG})$ Project ( 5X) and the Genetic Testing Reference Material Coordination Program ( $45 \mathrm{X})$, as well as high-depth short-read capture-based sequencing ( 330X), could not identify the 5-HTTLPR short (S) allele, nor could short-read sequencing phase any identified variants. In contrast, long-read SMRT sequencing unambiguously identified the 5-HTTLPR short (S) allele (frequency of 0.467) and phased SLC6A4 promoter haplotypes. Additionally, discordant rs 25531 genotypes were reviewed and determined to be short-read errors. Taken together, long-read SMRT sequencing is an innovative and robust method for phased resolution of the SLC6A4 promoter, which could enable more accurate pharmacogenetic testing for both research and clinical applications.
\end{abstract}

Keywords: SLC6A4; selective serotonin reuptake inhibitors (SSRI); long-read sequencing; single molecule real-time (SMRT) sequencing; Pacific Biosciences (PacBio); pharmacogenetics; pharmacogenomics; haplotype phasing

\section{Introduction}

Antidepressants are the third most commonly prescribed drug class in the United States, surpassed only by antihyperlipidemics and analgesics [1]. In addition to depression, selective serotonin reuptake inhibitors (SSRIs) are used to treat obsessive-compulsive disorder (OCD), panic disorder, posttraumatic stress disorder and anxiety disorders; however, efficacy among the different SSRIs has been estimated at only $\sim 65 \%$ across treated patients [2]. In an effort to improve antidepressant efficacy, pharmacogenetic studies have recently been undertaken to guide treatment based on 
common variants in genes involved in SSRI pharmacokinetics (e.g., CYP2D6 and CYP2C19) and pharmacodynamics (SLC6A4) [3].

The SLC6A4 gene at chromosome 17q11.2 encodes the serotonin (5-HT) transporter (5-HTT), which mediates 5-HT reuptake and is the major target of SSRI antidepressants. Expression of 5-HTT is directly correlated with 5-HT transporter function, which has been implicated as a susceptibility gene for several psychiatric disorders (e.g., affective disorders, schizophrenia, anxiety, autism, depression, suicide, OCD, and addiction [4]) as well as a pharmacogenetic determinant of interindividual SSRI response variability [5-8]. The polymorphic SLC6A4 promoter is composed of a variable number (11-24) of tandem repeat units (5-HTTLPR), which are 20-24 bp in length and highly homologous. The common 5-HTTLPR alleles implicated in SSRI response are the long (L; 16 repeats) and short (S; 14 repeats) haplotypes, which are defined by a $43 \mathrm{bp}$ insertion/deletion polymorphism (rs4795541) and are strongly associated with higher and lower gene expression, respectively [9-12]. In addition to these two alleles, extra-long (XL; 17-24 repeats) and extra-short (XS; 11-13 repeats) promoter alleles, have also been identified with low frequencies in the general population [13-18]; however, their effects on gene expression are still being elucidated [19].

In addition to the 5-HTTLPR $L>S$ variant (rs4795541), two SLC6A4 promoter sequence variants (rs25531 (c.-1936A >G), rs25532 (c.-1760C >T)) have also been reported to influence 5-HTT expression; however, they are most informative when considered as a haplotype with 5-HTTLPR [20,21]. The rs25531 $(A>G)$ variant is located in the sixth repeat unit and can occur on either the L or S 5-HTTLPR haplotype. Notably, when this variant is found on the L haplotype (" $\mathrm{L}_{\mathrm{G}}$ "), it is associated with lower gene expression, similar to the $S$ allele $[20,21]$. The rs $25532(C>T)$ variant is located $\sim 150$ bp from rs 25531 in the $\mu$ element. The presence of the $\mathrm{T}$ allele at this position also is associated with lower gene expression on both the L and S haplotypes [21]. Given the potential clinical significance of SLC6A4 and the importance of phasing multiple variants across the polymorphic repetitive promoter region, we developed a novel long-read single molecule real-time (SMRT) sequencing method to comprehensively characterize SLC6A4 promoter haplotypes.

\section{Materials and Methods}

\subsection{Samples and Publicly Available Data}

A total of 120 independent Coriell samples were subjected to long-read SMRT sequencing at the polymorphic SLC6A4 promoter region, primarily selected based on the availability of orthogonal short-read sequencing data. Among these samples, low-depth short-read genome sequencing (WGS) data ( 5X) was available for 32 samples from the 1000 Genomes (1KG) Project; high-depth short-read WGS data $(\sim 45 X)$ were available for 68 samples from the Centers for Disease Control and Genetic Testing Reference Material Coordination Program (GeT-RM) pharmacogenetic testing program [22]; and high-depth short-read capture-based panel data $(\sim 330 \mathrm{X})$ were available for 21 samples from an internal dataset. DNA samples were acquired from the Coriell Biorepository (Camden, NJ, USA), which are summarized in Supplemental Table S1.

1KG variant call format files (Phase 3 release v5a) were downloaded from the National Center for Biotechnology Information (NCBI) FTP server [23]. GeT-RM Illumina FASTQ and BAM files from were downloaded from the GeT-RM website and the European Nucleotide Archive (http://www.ebi.ac.uk/ena/data/view/PRJEB19931). 1KG Phase 3 and GeT-RM reference calls were evaluated using GATK Haplotype caller (version 4.1.3, -ERC GVCF -L 17:28563658-28564683), genotypes were called using PyPGx (v0.1.12, bam2vcf gatk 17:28563658-28564683 hg19) [24]. GeT-RM and high-depth short-read capture-based genotypes were also called using an internal Genome Analysis Tool Kit (GATK) best practices pipeline. GNU Parallel was used to increase the rate of data processing [25]. 


\subsection{PCR Amplification of the SLC6A4 Promoter}

Four overlapping primer sets (with a common forward primer) were used to amplify the SLC6A4 promoter region (Table 1), which included universal oligonucleotide tags for subsequent barcoding and SMRT sequencing. Long-range PCR reactions were performed in $20 \mu \mathrm{L}$ containing $100 \mathrm{ng}$ of DNA, 1X SequalPrep ${ }^{\mathrm{TM}}$ Reaction buffer (Invitrogen, Carlsbad, CA, USA), $0.5 \mu \mathrm{M}$ of forward and reverse primers, and 1.8 units of SequalPrep ${ }^{\mathrm{TM}}$ polymerase. Amplification conditions were identical for all primer sets and consisted of an initial denaturation step at $94^{\circ} \mathrm{C}$ for $2 \mathrm{~min}$ followed by 10 amplification cycles $\left(94^{\circ} \mathrm{C}\right.$ for $10 \mathrm{~s}, 63.6^{\circ} \mathrm{C}$ for $30 \mathrm{~s}$, and $68^{\circ} \mathrm{C}$ for $\left.90 \mathrm{~s}\right)$, another 20 amplification cycles $\left(94^{\circ} \mathrm{C}\right.$ for $10 \mathrm{~s}$, $63.6^{\circ} \mathrm{C}$ for $30 \mathrm{~s}$, and $68^{\circ} \mathrm{C}$ for $90 \mathrm{~s}+20 \mathrm{~s} / \mathrm{cycle}$ ), and a final extension at $72{ }^{\circ} \mathrm{C}$ for $5 \mathrm{~min}$. These products were used as templates for barcoding prior to SMRT sequencing.

Table 1. Primer sequences used to amplify the SLC6A4 promoter region.

\begin{tabular}{|c|c|c|}
\hline Primer ID & Primer Sequence * & Amplicon Size (bp) \\
\hline SLC6A4F & $\begin{array}{c}\text { Forward primer } \\
5^{\prime} \text {-ATGGGTTCCAGAGTCAATCCTTTGCGTTTTCTGTTGCCCT-3' } \\
\text { Reverse primers }\end{array}$ & - \\
\hline SLC6A4R1 & 5'-GAAAGGTCTGGAGTCTTGATGAGGGACTGAGCTGGACAACCAC-3' & 699 \\
\hline SLC6A4R2 & 5'-GAAAGGTCTGGAGTCTTGATCCCAGCAGGAGCCTATTGTT-3' & 1026 \\
\hline SLC6A4R3 & 5'-GAAAGGTCTGGAGTCTTGATTCTCTTGACCTCGGACACCT-3' & 1536 \\
\hline SLC6A4R4 & $5^{\prime}$-GAAAGGTCTGGAGTCTTGATGAAAGAAACGTGGGTTCGAGG-3' & 2096 \\
\hline
\end{tabular}

${ }^{*}$ Universal oligonucleotide tag sequence nucleotides are underlined.

\subsection{SLC6A4 Promoter Sample Barcoding and Pooling}

First round amplification products were used as template for a subsequent PCR that incorporated forward and reverse barcodes using forward (5'-[barcode]-ATGGGTTCCAGAGTCAATC- $3^{\prime}$ ) and reverse (5'-[barcode]-GAAAGGTCTGGAGTCTTGAT-3') primers (Supplemental Table S2). The PCR conditions for this reaction were identical to the first round PCR described above. Agencourt ${ }^{\circledR}$ AMPure ${ }^{\circledR}$ XP beads were used to purify all barcoded PCR amplicons, which were quantified by Nanodrop 1000. Following purification and quantitation, sample library pools were generated with equal molecule quantities of PCR amplicons using the following formula to calculate the required volume of each amplicon [26]:

$$
V(i)=\frac{M}{n \times C(i)}
$$

where $M$ is the total mass of pooled PCR amplicons, $n$ is the total number of samples, $V(i)$ is the volume of each PCR amplicon, and C(i) is the concentration of each amplicon. A total of $4000 \mathrm{ng}$ of pooled PCR amplicons was submitted for SMRT sequencing.

\subsection{Single Molecule Real-Time (SMRT) Sequencing}

SMRT sequencing was executed according to the P6-C4 Pacific Biosciences protocol with a movie collection time of $180 \mathrm{~min}$ on the Sequel instrument, as per the manufacturer's instructions and as previously described [26]. SMRT sequencing analysis included demultiplexing, alignment, quality score recalibration, and variant calling. Raw sequencing data in FASTQ format were demultiplexed using NGSutils, sequencing reads were aligned using BWA-MEM, and variant calling was performed using GATK [26].

\subsection{Sanger Sequencing Confirmation}

Selected samples were validated by orthogonal Sanger sequencing using the following primer set: FWD 5'-CTTTGCGTTTTCTGTTGCCCT-3'; REV 5'-CCCAGCAGGAGCCTATTGTT-3'. These primers generated a $1026 \mathrm{bp}$ amplicon using the same PCR conditions described above. PCR fragments 
were analyzed using the Applied Biosystems 3730xl DNA Analyzer, and chromatogram traces were evaluated using NG_011747.2 as a reference sequence and Chromas v2.6.6 (South Brisbane, Australia).

\section{Results}

\subsection{SLC6A4 Promoter Short-Read Sequencing}

Genotype results from the interrogated SLC6A4 promoter region identified by short-read WGS (1KG and GeT-RM) and capture-based enrichment sequencing are summarized in Supplemental Table S2. The 5-HTTLPR (rs4795541), rs25531 and rs25532 variant sites were either not detectable or incorrectly genotyped among the 1KG WGS cohort, the GeT-RM WGS cohort, and the short-read capture-based sequencing cohort in 32/32 (100\%), 60/68 (88\%) and 17/21 (81\%) samples and 87/96 (91\%), $85 / 204(42 \%)$ and 34/63 (54\%) variant sites, respectively (Supplemental Table S2).

\subsection{SLC6A4 Promoter Long-Read SMRT Sequencing}

All 120 samples were subjected to long-read SMRT sequencing across the polymorphic SLC6A4 promoter, and a representative sample with both short- and long-read sequencing data available is illustrated in Figure 1. Long-read SMRT sequencing results of additional SLC6A4 diplotypes are illustrated in Figure 2. In contrast to the short-read sequencing datasets, the 5-HTTLPR short (S) allele (rs4795541) was detected by long-read SMRT sequencing in 79 samples, resulting in a MAF of 0.467 (112/240). In addition, the SLC6A4 rs25531 and rs25532 variants were detectable in all samples and their MAFs were 0.113 (27/240) and 0.088 (21/240), respectively. In addition, long-read SMRT sequencing detected two samples with extra-long (XL) 5-HTTLPR alleles that were not detected by short-read sequencing. Six additional SLC6A4 promoter sequence variants were also detected and phased by long-read SMRT sequencing (Supplemental Table S3), which were inconsistently detected among the short-read sequencing datasets.

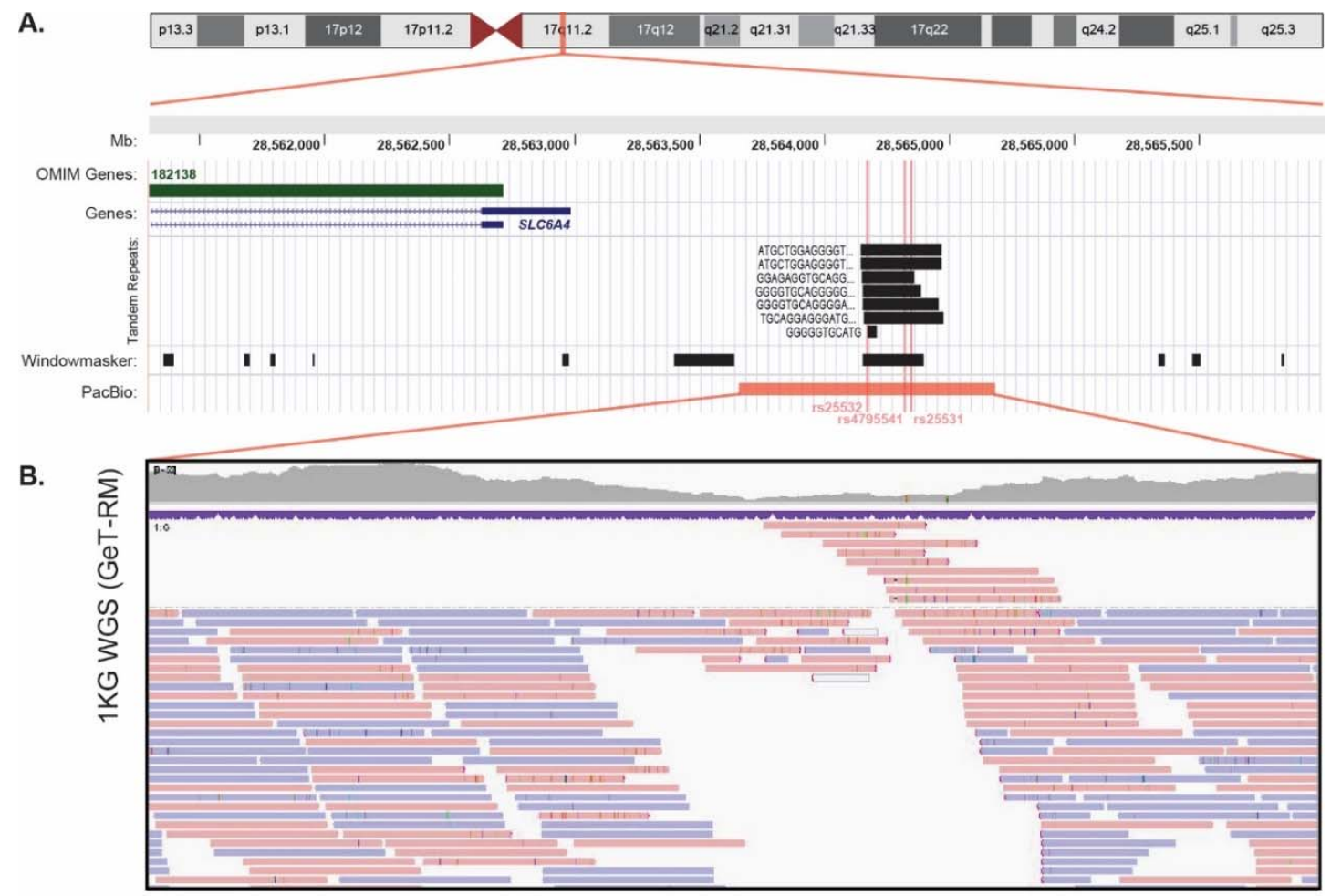

Figure 1. Cont. 
C.

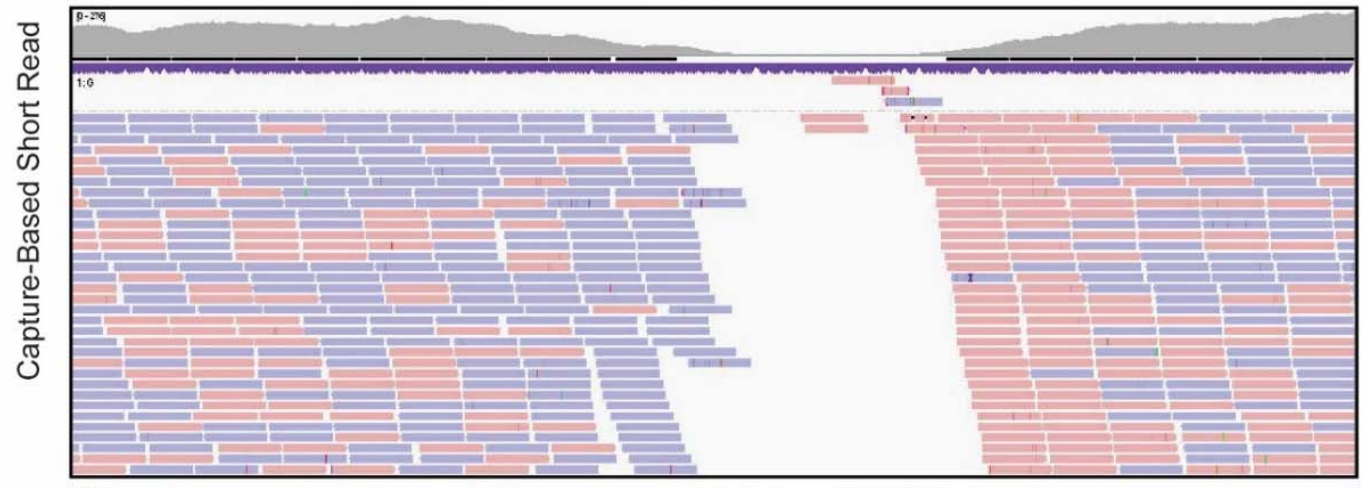

D.

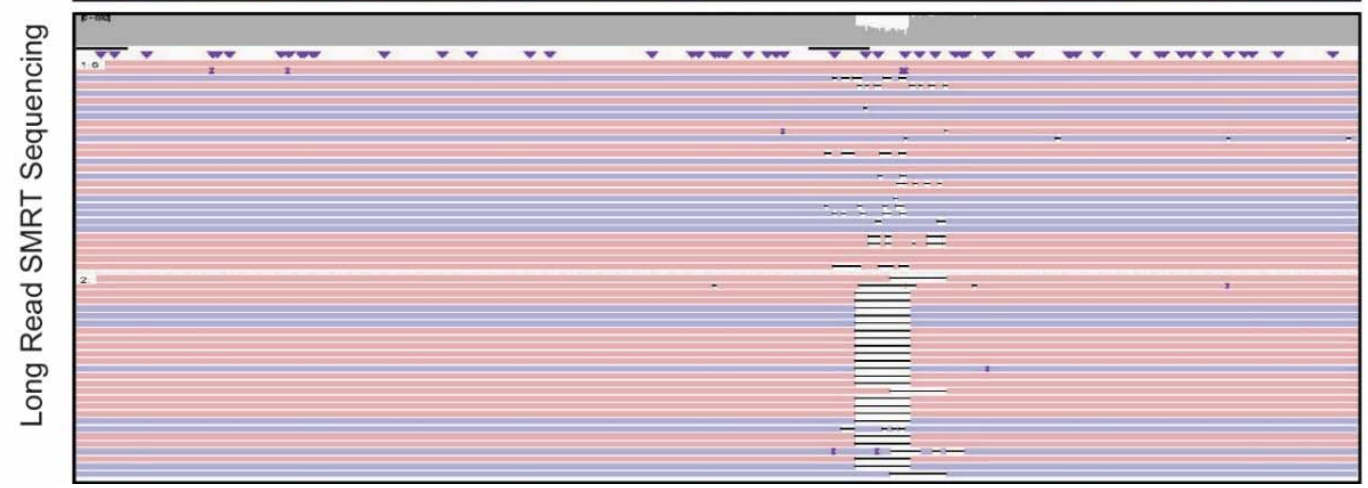

Figure 1. Representative SLC6A4 promoter sequencing of the NA12156 Coriell sample using short-read (Genetic Testing Reference Material Coordination Program (GeT-RM) genome sequencing (WGS), and capture-based sequencing) and long-read single molecule real-time (SMRT) sequencing. Note the inability to interrogate the repetitive SLC6A4 promoter region (A) by two short-read sequencing approaches (WGS (B) and capture-based sequencing (C)), in contrast to the 5-HTTLPR short (S) allele detection and haplotype phasing accomplished by long-read SMRT sequencing (D).

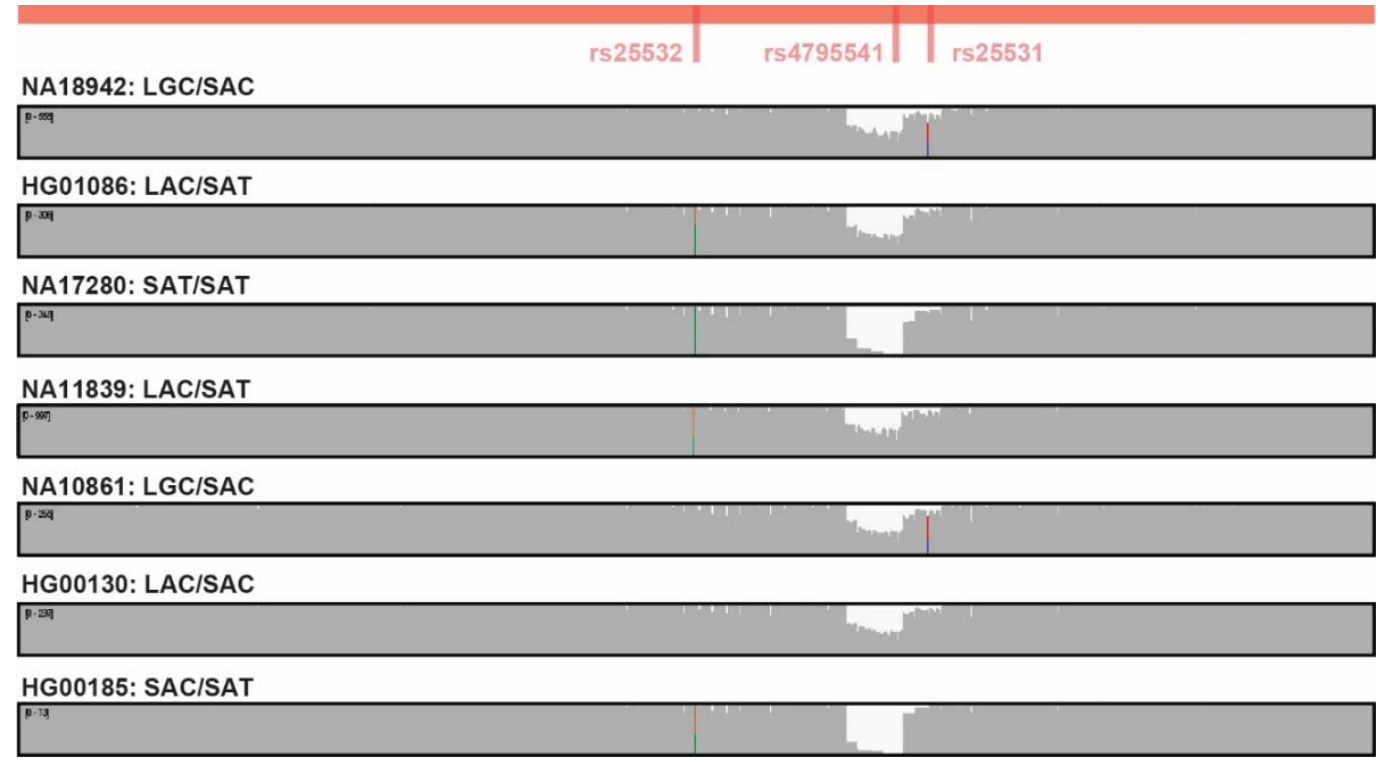

Figure 2. Representative SLC6A4 promoter sequencing results from seven Coriell DNA samples using long-read SMRT sequencing: NA18942 (LGC/SAC), HG01086 (LAC/SAT), NA17280 SAT/SAT, NA11839 (LAC/SAT), NA10861 (LGC/SAC), HG00130 (LAC/SAC), HG00185 (SAC/SAT).

In addition to being more accurate at this locus than short-read sequencing, long-read SMRT sequencing also unambiguously phased the polymorphic SLC6A4 promoter in all samples, including complex compound heterozygous diplotypes (Figure 2). The three clinically significant 
SLC6A4 promoter variants (5-HTTLPR (rs4795541), rs25531, rs25532) were phased and the identified diplotypes and haplotype frequencies are summarized in Supplemental Table S2 and Table 2 , respectively.

Table 2. SLC6A4 promoter haplotypes detected by SMRT sequencing.

\begin{tabular}{ccccccc}
\hline & LAC & SAC & LGC & SAT & XLAC & SGC \\
\hline$n$ freq $) *^{*}$ & $101(0.421)$ & $89(0.371)$ & $26(0.108)$ & $21(0.088)$ & $2(0.008)$ & $1(0.004)$ \\
\hline
\end{tabular}

${ }^{*}$ Total $n=240$ alleles. The haplotypes are defined by the rs4795541 (L/S)/rs25531 (A/G)/rs25532 (C/T) variants.

\subsection{SLC6A4 Promoter Long-Read SMRT Sequencing Sanger Validation}

Accuracy of long-read SMRT sequencing at the polymorphic SLC6A4 promoter was further evaluated by orthogonal Sanger sequencing. Six DNA samples were selected based on their representative diplotypes: LAC/LGC (HG00276, NA12336), LGC/SAC (HG01190), LAC/SAT (NA07000), LAC/XLAC (NA18855), and SAC/XLAC (NA19174). Sanger sequencing confirmed all variant alleles identified by long-read SMRT sequencing, including the short (S) and extra-long (XL) 5-HTTLPR alleles that were not detected by short-read sequencing (Supplemental Table S4 and Figure S1).

\subsection{SLC6A4 Promoter Long-Read SMRT Sequencing Precision}

Precision of long-read SMRT sequencing was measured by subjecting reference material samples with identified SLC6A4 variant alleles ( $n=15)$ to intra-run (repeatability) and inter-run (reproducibility) triplicate testing (i.e., 3:1:1 validation). In summary, the intra- and inter-run genotype and diplotype concordances for the 15 control samples were both 100\% (225/225 genotypes; 150/150 haplotypes) (Supplemental Table S5).

\section{Discussion}

Given the emerging clinical significance of SLC6A4 in interindividual SSRI response variability and the repetitive architecture of the homologous $S L C 6 A 4$ promoter, we developed a novel long-read SMRT sequencing assay using the PacBio platform to comprehensively characterize SLC6A4 promoter region haplotypes. Our innovative method enabled the phased resolution of complex SLC6A4 promoter diplotypes, which was not possible using short-read WGS data $(\sim 5 \mathrm{X}$ and $\sim 45 \mathrm{X})$ or high-depth capture-based short-read sequencing data ( 330X). This novel pharmacogenetic application of long-read SMRT sequencing adds to previously reported methods that employed the PacBio platform to interrogate clinically relevant low-complexity homologous regions of the human genome [26,27].

The SLC6A4 gene encodes 5-HTT, which plays a key role in the central nervous system by regulating serotonergic signaling via transport of 5-HT from the synaptic cleft back into the pre-synaptic terminal for re-utilization. As such, SLC6A4 has previously been associated with a range of behavioral and psychiatric disorders including depression, OCD, anxiety and schizophrenia [4]. Consistent with the role of 5-HTT in psychiatric phenotypes, variant SLC6A4 alleles have also been implicated in interindividual SSRI response [5-8]. The principal SLC6A4 variant associated with antidepressant efficacy is the 5-HTTLPR insertion/deletion polymorphism (rs4795541), which is located in the SLC6A4 promoter and regulates gene expression. However, this region is challenging to interrogate given that the promoter is a GC rich variable number tandem repeat (VNTR) comprised of 11 to 24 homologous units.

The commonly studied 5-HTTLPR long (L) and short (S) alleles have variable frequencies across ancestral populations. Specifically, the 5-HTTLPR short (S) allele has a higher frequency in Asian populations (55-82\%) [28], and a frequency in Europeans and Africans of 35-50\% [28] and $22-25 \%[29,30]$, respectively. Although our study was not intended to measure population frequencies of the 5-HTTLPR L $>$ S insertion/deletion polymorphism, long-read SMRT sequencing of 120 reference material samples detected a minor allele (S) frequency of $46.7 \%$, which is consistent with a general population frequency. 
In addition to 5-HTTLPR, the neighboring rs25531 (c.-1936A>G) variant has also been associated with reduced SLC6A4 expression. Importantly, individuals who carry the 5-HTTLPR long (L) allele also have reduced SLC6A4 expression when they concurrently harbor rs25531 c.-1936G [20,21], underscoring the importance of phasing SLC6A4 variants into promoter haplotypes. Of note, the overall MAF of rs25531 in the gnomAD database is 0.1706 [31]; however, it is annotated as a low complexity region with a low genotype quality metric, and is only covered in fewer than $50 \%$ of individuals in gnomAD v2.1.1.

In addition to 5-HTTLPR and rs25531, rs25532 (c.-1760C > T) has also emerged as an independent determinant of SLC6A4 expression. Notably, the rs25532 c.-1760T allele has been correlated with a $15-80 \%$ reduction in SLC6A4 gene expression depending on the presence of other variants within the promoter haplotype [21]. Although the independent influence of rs25532 on SLC6A4 expression is significant, it is most informative when taken into consideration as a haplotype with 5-HTTLPR and rs25531 [32]. The overall MAF of rs25532 in the gnomAD database is 0.0683 [31]; however, it is also annotated as a low complexity region. Interestingly, our analysis of 120 samples by long-read SMRT sequencing did not identify any individuals with the rs25532 c.-1760T allele in cis with the rs25531 c.-1936G allele, which is consistent with previous studies that suggested rs25532 c.-1760T is in linkage disequilibrium with rs25531 c.-1936A [21].

Short-read sequencing is not effective at accurately interrogating the SLC6A4 promoter, particularly across the VNTR that includes the 5-HTTLPR insertion/deletion (L $>$ S) polymorphism. This overarching limitation of short-read sequencing has previously been acknowledged, as low complexity regions and tandem repeats in the human genome are notoriously challenging for short-read platforms [33]. However, the ongoing improvements in long-read sequencing chemistry and throughput are increasingly enabling more accurate interrogation of these difficult regions for both research and clinical applications [34]. Our SLC6A4 long-read SMRT sequencing results further support the integration of third-generation sequencing platforms for more accurate and comprehensive characterization of low-complexity pharmacogenetic regions.

In addition to interrogating homologous regions, another advantage of long-read SMRT sequencing is the ability to phase variants and define diplotypes over kilobases of genomic sequence. We previously leveraged long-read SMRT sequencing for full-length characterization of the CYP2D6 gene [26], which led to the unambiguous detection of phased diplotypes and inferred phenotypes. Variant phasing is critical for more informed classification and interpretation in medical genetics, and defining full-gene haplotypes is an integral component of pharmacogenetics. The star $\left(^{*}\right)$ allele nomenclature system was developed over 20 years ago as a mechanism to characterize and report pharmacogenetic haplotypes, which is now centralized and administered by the NIH-funded Pharmacogene Variation (PharmVar) Consortium (https://www.pharmvar.org/) [35]. Although star $\left(^{*}\right)$ alleles have not yet been defined for SLC6A4, our long-read SMRT sequencing assay efficiently phased clinically relevant variants across the polymorphic homologous SLC6A4 promoter, including the rare extra-long (XL) allele.

\section{Conclusions}

In conclusion, SLC6A4 long-read SMRT sequencing is a reliable and validated third-generation sequencing technique that can accurately interrogate the low-complexity homologous SLC6A4 promoter region. This novel method adds to previously reported long-read SMRT sequencing applications in pharmacogenomics, underscoring its utility for high-throughput variant detection and haplotype phasing. The capability to detect and phase the 5-HTTLPR L $>$ S (rs4795541), rs25531 (c.-1936A>G), and rs 25532 (c. $-1760 \mathrm{C}>\mathrm{T}$ ) variants in the polymorphic SLC6A4 promoter indicates that this method likely will have utility for both research and clinical testing applications. 
Supplementary Materials: The following are available online at http://www.mdpi.com/2073-4425/11/11/1333/s1. Table S1: Summary of SLC6A4 Samples and Datasets. Table S2: Summary of SLC6A4 Promoter Sequencing Results. Table S3: Summary of Additional SLC6A4 Promoter Variants Detected by Long-Read SMRT Sequencing. Table S4: SLC6A4 Promoter Sanger Sequencing Confirmation. Table S5: Summary of SLC6A4 Long-Read SMRT Sequencing Precision. Figure S1: Representative Sanger sequencing results from selected SLC6A4promoter reference material samples.

Author Contributions: Conceptualization, M.R.B., Y.Y., S.A.S.; methodology, M.R.B., Y.Y., E.R.S., S.A.S.; software, Y.Y., E.R.S.; validation, M.R.B., Y.Y.; formal analysis, M.R.B., S.A.S.; investigation, M.R.B., Y.Y., E.R.S., S.A.S.; resources, R.J.D., S.A.S.; data curation, M.R.B., Y.Y., E.R.S.; writing-original draft preparation, M.R.B., S.A.S.; writing—review and editing, M.R.B., Y.Y., E.R.S., R.J.D., S.A.S.; visualization, Y.Y., S.A.S.; supervision, S.A.S. All authors have read and agreed to the published version of the manuscript.

Funding: This research received no external funding.

Acknowledgments: The authors would like to thank Melissa L. Smith at the Icahn School of Medicine at Mount Sinai, for technical consultation during the study.

Conflicts of Interest: S.A.S. is a consultant for Sema4, Stamford, CT; no other authors report any conflict of interest.

\section{References}

1. National Center for Health Statistics. Health, United States, 2016: With Chartbook on Long-Term Trends in Health; National Center for Health Statistics: Hyattsville, MD, USA, 2017.

2. Sinyor, M.; Levitt, A.J.; Cheung, A.H.; Schaffer, A.; Kiss, A.; Dowlati, Y.; Lanctôt, K.L. Does inclusion of a placebo arm influence response to active antidepressant treatment in randomized controlled trials? Results from pooled and meta-analyses. J. Clin. Psychiatry 2010, 71, 270-279. [CrossRef] [PubMed]

3. Pérez, V.; Salavert, A.; Espadaler, J.; Tuson, M.; Saiz-Ruiz, J.; Sáez-Navarro, C.; Bobes, J.; Baca-García, E.; Vieta, E.; Olivares, J.M.; et al. Efficacy of prospective pharmacogenetic testing in the treatment of major depressive disorder: Results of a randomized, double-blind clinical trial. BMC Psychiatry 2017, 17, 250. [CrossRef] [PubMed]

4. Haddley, K.; Bubb, V.J.; Breen, G.; Parades-Esquivel, U.M.; Quinn, J.P. Behavioural genetics of the serotonin transporter. Curr. Top Behav. Neurosci. 2012, 12, 503-535. [CrossRef] [PubMed]

5. Hu, X.Z.; Rush, A.J.; Charney, D.; Wilson, A.F.; Sorant, A.J.; Papanicolaou, G.J.; Fava, M.; Trivedi, M.H.; Wisniewski, S.R.; Laje, G.; et al. Association between a functional serotonin transporter promoter polymorphism and citalopram treatment in adult outpatients with major depression. Arch. Gen. Psychiatry 2007, 64, 783-792. [CrossRef] [PubMed]

6. Masoliver, E.; Menoyo, A.; Pérez, V.; Volpini, V.; Rio, E.D.; Pérez, J.; Alvarez, E.; Baiget, M. Serotonin transporter linked promoter (polymorphism) in the serotonin transporter gene may be associated with antidepressant-induced mania in bipolar disorder. Psychiatr. Genet. 2006, 16, 25-29. [CrossRef] [PubMed]

7. Perlis, R.H.; Mischoulon, D.; Smoller, J.W.; Wan, Y.J.; Lamon-Fava, S.; Lin, K.M.; Rosenbaum, J.F.; Fava, M. Serotonin transporter polymorphisms and adverse effects with fluoxetine treatment. Biol. Psychiatry 2003, 54, 879-883. [CrossRef]

8. Putzhammer, A.; Schoeler, A.; Rohrmeier, T.; Sand, P.; Hajak, G.; Eichhammer, P. Evidence of a role for the 5-HTTLPR genotype in the modulation of motor response to antidepressant treatment. Psychopharmacology 2005, 178, 303-308. [CrossRef]

9. Heils, A.; Teufel, A.; Petri, S.; Seemann, M.; Bengel, D.; Balling, U.; Riederer, P.; Lesch, K.P. Functional promoter and polyadenylation site mapping of the human serotonin (5-HT) transporter gene. J. Neural Transm. Gen. Sect. 1995, 102, 247-254. [CrossRef]

10. Heils, A.; Teufel, A.; Petri, S.; Stöber, G.; Riederer, P.; Bengel, D.; Lesch, K.P. Allelic variation of human serotonin transporter gene expression. J. Neurochem. 1996, 66, 2621-2624. [CrossRef]

11. Lesch, K.P.; Bengel, D.; Heils, A.; Sabol, S.Z.; Greenberg, B.D.; Petri, S.; Benjamin, J.; Müller, C.R.; Hamer, D.H.; Murphy, D.L. Association of anxiety-related traits with a polymorphism in the serotonin transporter gene regulatory region. Science 1996, 274, 1527-1531. [CrossRef]

12. Mortensen, O.V.; Thomassen, M.; Larsen, M.B.; Whittemore, S.R.; Wiborg, O. Functional analysis of a novel human serotonin transporter gene promoter in immortalized raphe cells. Brain Res. Mol. Brain Res. 1999, 68, 141-148. [CrossRef] 
13. Delbrück, S.J.; Wendel, B.; Grunewald, I.; Sander, T.; Morris-Rosendahl, D.; Crocq, M.A.; Berrettini, W.H.; Hoehe, M.R. A novel allelic variant of the human serotonin transporter gene regulatory polymorphism. Cytogenet. Cell Genet. 1997, 79, 214-220. [CrossRef] [PubMed]

14. Frisch, A.; Finkel, B.; Michaelovsky, E.; Sigal, M.; Laor, N.; Weizman, R. A rare short allele of the serotonin transporter promoter region (5-HTTLPR) found in an aggressive schizophrenic patient of Jewish Libyan origin. Psychiatr. Genet. 2000, 10, 179-183. [CrossRef] [PubMed]

15. Gelernter, J.; Kranzler, H.; Cubells, J.F. Serotonin transporter protein (SLC6A4) allele and haplotype frequencies and linkage disequilibria in African- and European-American and Japanese populations and in alcohol-dependent subjects. Hum. Genet. 1997, 101, 243-246. [CrossRef] [PubMed]

16. Murdoch, J.D.; Speed, W.C.; Pakstis, A.J.; Heffelfinger, C.E.; Kidd, K.K. Worldwide population variation and haplotype analysis at the serotonin transporter gene SLC6A4 and implications for association studies. Biol. Psychiatry 2013, 74, 879-889. [CrossRef] [PubMed]

17. Nakamura, M.; Ueno, S.; Sano, A.; Tanabe, H. The human serotonin transporter gene linked polymorphism (5-HTTLPR) shows ten novel allelic variants. Mol. Psychiatry 2000, 5, 32-38. [CrossRef]

18. Vijayendran, M.; Cutrona, C.; Beach, S.R.; Brody, G.H.; Russell, D.; Philibert, R.A. The relationship of the serotonin transporter (SLC6A4) extra long variant to gene expression in an African American sample. Am. J. Med. Genet. B Neuropsychiatr. Genet. 2012, 159B, 611-612. [CrossRef]

19. Ehli, E.A.; Hu, Y.; Lengyel-Nelson, T.; Hudziak, J.J.; Davies, G.E. Identification and functional characterization of three novel alleles for the serotonin transporter-linked polymorphic region. Mol. Psychiatry 2012, 17, 185-192. [CrossRef]

20. Hu, X.Z.; Lipsky, R.H.; Zhu, G.; Akhtar, L.A.; Taubman, J.; Greenberg, B.D.; Xu, K.; Arnold, P.D.; Richter, M.A.; Kennedy, J.L.; et al. Serotonin transporter promoter gain-of-function genotypes are linked to obsessive-compulsive disorder. Am. J. Hum. Genet. 2006, 78, 815-826. [CrossRef]

21. Wendland, J.R.; Moya, P.R.; Kruse, M.R.; Ren-Patterson, R.F.; Jensen, C.L.; Timpano, K.R.; Murphy, D.L. A novel, putative gain-of-function haplotype at SLC6A4 associates with obsessive-compulsive disorder. Hum. Mol. Genet. 2008, 17, 717-723. [CrossRef]

22. Pratt, V.M.; Everts, R.E.; Aggarwal, P.; Beyer, B.N.; Broeckel, U.; Epstein-Baak, R.; Hujsak, P.; Kornreich, R.; Liao, J.; Lorier, R.; et al. Characterization of 137 Genomic DNA Reference Materials for 28 Pharmacogenetic Genes: A GeT-RM Collaborative Project. J. Mol. Diagn. 2016, 18, 109-123. [CrossRef] [PubMed]

23. Genomes Project, C.; Auton, A.; Brooks, L.D.; Durbin, R.M.; Garrison, E.P.; Kang, H.M.; Korbel, J.O.; Marchini, J.L.; McCarthy, S.; McVean, G.A.; et al. A global reference for human genetic variation. Nature 2015, 526, 68-74. [CrossRef]

24. PyPGx. Available online: https://github.com/sbslee/pypgx (accessed on 11 November 2020).

25. Tange, O. GNU Parallel 2018. 2018. Available online: http://doi.org/10.5281/zenodo.1146014 (accessed on 11 November 2020).

26. Qiao, W.; Yang, Y.; Sebra, R.; Mendiratta, G.; Gaedigk, A.; Desnick, R.J.; Scott, S.A. Long-Read Single Molecule Real-Time Full Gene Sequencing of Cytochrome P450-2D6. Hum. Mutat. 2016, 37, 315-323. [CrossRef] [PubMed]

27. Borras, D.M.; Vossen, R.; Liem, M.; Buermans, H.P.J.; Dauwerse, H.; van Heusden, D.; Gansevoort, R.T.; den Dunnen, J.T.; Janssen, B.; Peters, D.J.M.; et al. Detecting PKD1 variants in polycystic kidney disease patients by single-molecule long-read sequencing. Hum. Mutat. 2017, 38, 870-879. [CrossRef]

28. Li, H.; Li, S.; Wang, Q.; Pan, L.; Jiang, F.; Yang, X.; Zhang, N.; Han, M.; Jia, C. Association of 5-HTTLPR polymorphism with smoking behaviors: A meta-analysis. Physiol. Behav. 2015, 152, 32-40. [CrossRef]

29. Kranzler, H.R.; Scott, D.; Tennen, H.; Feinn, R.; Williams, C.; Armeli, S.; Taylor, R.E.; Briggs-Gowan, M.J.; Covault, J. The 5-HTTLPR polymorphism moderates the effect of stressful life events on drinking behavior in college students of African descent. Am. J. Med. Genet. B Neuropsychiatr. Genet. 2012, 159B, 484-490. [CrossRef]

30. Arieff, Z.; Kaur, M.; Gameeldien, H.; van der Merwe, L.; Bajic, V.B. 5-HTTLPR polymorphism: Analysis in South African autistic individuals. Hum. Biol. 2010, 82, 291-300. [CrossRef]

31. Karczewski, K.J.; Francioli, L.C.; Tiao, G.; Cummings, B.B.; Alfoldi, J.; Wang, Q.; Collins, R.L.; Laricchia, K.M.; Ganna, A.; Birnbaum, D.P.; et al. The mutational constraint spectrum quantified from variation in 141,456 humans. Nature 2020, 581, 434-443. [CrossRef] 
32. Iurescia, S.; Seripa, D.; Rinaldi, M. Role of the 5-HTTLPR and SNP Promoter Polymorphisms on Serotonin Transporter Gene Expression: A Closer Look at Genetic Architecture and In Vitro Functional Studies of Common and Uncommon Allelic Variants. Mol. Neurobiol. 2016, 53, 5510-5526. [CrossRef]

33. Mandelker, D.; Schmidt, R.J.; Ankala, A.; McDonald Gibson, K.; Bowser, M.; Sharma, H.; Duffy, E.; Hegde, M.; Santani, A.; Lebo, M.; et al. Navigating highly homologous genes in a molecular diagnostic setting: A resource for clinical next-generation sequencing. Genet. Med. 2016, 18, 1282-1289. [CrossRef]

34. Ardui, S.; Ameur, A.; Vermeesch, J.R.; Hestand, M.S. Single molecule real-time (SMRT) sequencing comes of age: Applications and utilities for medical diagnostics. Nucleic Acids Res. 2018, 46, 2159-2168. [CrossRef] [PubMed]

35. Gaedigk, A.; Sangkuhl, K.; Whirl-Carrillo, M.; Twist, G.P.; Klein, T.E.; Miller, N.A.; PharmVar Steering, C. The Evolution of PharmVar. Clin. Pharmacol. Ther. 2019, 105, 29-32. [CrossRef] [PubMed]

Publisher's Note: MDPI stays neutral with regard to jurisdictional claims in published maps and institutional affiliations.

(C) 2020 by the authors. Licensee MDPI, Basel, Switzerland. This article is an open access article distributed under the terms and conditions of the Creative Commons Attribution (CC BY) license (http://creativecommons.org/licenses/by/4.0/). 